\title{
MÉTODOS DE CONTROLE DO FLUXO DE ENTRADA PARA GRAFOS DE EVENTOS TEMPORIZADOS
}

\author{
Dias, J. R. S.*, MaiA, C. A..$^{\dagger}$ LuCEnA JR, V. F. ${ }^{\ddagger}$ \\ *Programa de Pós-Graduação em Engenharia Elétrica - Universidade Federal de Minas Gerais \\ Av. Antônio Carlos 6627, 31270-901 \\ Belo Horizonte, MG, Brasil \\ $\dagger^{\dagger}$ Depto. Engenharia Elétrica - UFMG \\ Av. Antônio Carlos 6627 - Pampulha \\ Belo Horizonte, Minas Gerais, Brasil \\ $\ddagger$ Depto. Engenharia Elétrica - UFAM \\ Av. General Rodrigo Otávio Jordão Ramos, 3000 - Coroado \\ Manaus , Amazonas, Brasil
}

Emails: ricardodias@ifam.edu.br, maia@cpdee.ufmg.br, lucena@ufam.edu.br

\begin{abstract}
This paper deals with feedback controller synthesis for timed event graphs in max-plus linear systems. In particular, will be analyzed two methods to calculate a controller that controls the flow of material in a production system. The principal aim is minimize inventory system meeting the demand specifications, so that the controller has the "best" possible control. The best possible control is the one that slows the maximum the firing of input events into the system, delaying the maximum input materials in the process. The synthesis presented here is mainly based on residuation theory results.
\end{abstract}

Keywords_ Discrete Events Systems, Control Systems, Max-plus Algebra.

Resumo - Este artigo trata da análise e síntese de controladores de realimentação para grafos de eventos temporizados em sistemas lineares max-plus. Em particular, serão analisados dois métodos para calcular os parâmetros de um controlador que controla o fluxo de material de entrada em um sistema de produção. O principal objetivo é otimizar o fluxo de entrada de material, satisfazendo as especificações de demanda, de modo que o controlador tenha o "melhor controle possível". O melhor controle possível é o que retarda ao máximo o disparo de eventos de entrada no sistema, atrasando ao máximo a entrada de materiais no processo. A síntese aqui apresentada é com base nos resultados da teoria residuação.

Palavras-chave_- Sistemas a Eventos Discretos, Controle de Sistemas, Álgebra Max-plus.

\section{Introdução}

Em um sistema de manufatura, como em qualquer outro tipo de sistema, existem muitas atividades e operações que podem ser controladas e automatizadas. Para cada tipo de controle proposto existe uma técnica específica para ser aplicada. Casos particulares de soluções são propostos para resolver a maioria dos problemas em um sistema industrial.

Os principais objetivos relacionados com a automatização da produção estão relacionados com a redução do tempo de produção, eliminação dos problemas durante o processo e redução das perdas. Empregar o melhor método para cada tipo de problema é essencial para atingir o resultado final desejado.

Existem muitas técnicas para modelagem de Sistemas a Eventos Discretos (SED). Uma maneira formal de descrever um SED é através dos grafos de eventos temporizados (GET's), dentre esses sistemas encontram-se os sistemas de manufatura. Os GET's são uma subclasse de Redes de Petri. Em um GET, o mecanismo de temporização está relacionado com o tempo de permanência das fichas nos lugares da rede. Nele, todos os lugares possuem um único arco de entrada e um único arco de saída (Murata, 1989), (Baccelli et al., 1992). O comportamento dinâmico do GET pode ser descrito por meio de equações de datadores, e essas equações pedem ser operacionalizadas de acordo com a álgebra max-plus. As equações são obtidas de forma recursiva.

Os problemas de controle para GET's são usualmente propostos utilizando-se a política de gestão Just-in-Time, a qual objetiva minimizar os estoques do sistema atendendo as especificações de demanda. De um modo geral, as propostas de controle estão baseadas no controle em malha aberta, utilizando-se a pré-compensação, e em malha fechada, utilizando-se a realimentação (Maia, 2003).

Alguns trabalhos relacionados ao controle para GET's tratam da modelagem de sistemas que podem ser caracterizados como sistemas a eventos discretos que são caracterizados por fenômenos de sincronização e de atraso. Boimond and Ferrier (1996) propõe uma estrutura de controle interno, utilizado na teoria de controle convencional, para projetar um controlador que trabalha como o modelo inverso do sistema. Cottenceau et al. (1999) trata de um sistema de realimenta- 
ção que atrasa ao máximo a entrada do sistema, mantendo a mesma relação de transferência entre a entrada e a saída. Menguy et al. (2000) aborda o controle just-in-time para sistemas com entradas controladas ou não-controladas. Schutter and van den Boom (2000) adota uma estratégia de controle baseada na abordagem por modelo de referência. Cottenceau et al. (2001) trata da síntese de controle de realimentação de GET's. Maia et al. (2003) apresenta diversas estratégias de controle baseadas na abordagem por modelo de referência. Zhu et al. (2004) visa desenvolver um método de agendamento de tarefas para uma célula de manufatura flexível por meio da fusão das redes de Petri temporizadas e da álgebra maxplus. Brunsch et al. (2012) trata da modelagem e controle de sistemas HTS(high-throughput screening), calculando um modelo de controlador para lidar com desvios inesperados da operação cíclica pré-determinada durante a execução do sistema.

A principal contribuição deste trabalho é propor métodos para o controle do fluxo de entrada de sistemas modelados por grafos de eventos temporizados, utilizando-se a base em dióides $\overline{\mathbb{Z}}_{\max }$.

Os métodos do caminho crítico e o método para sincronização dos disparos de duas transições internas foram desenvolvidos pelos autores deste artigo. A aplicação do primeiro método já foi desenvolvida para sistemas com múltiplas entradas e uma única saída (Dias et al., 2012), neste artigo será aplicado para sistemas com múltiplas entradas e múltiplas saídas. Uma versão estendida do segundo método está em análise para publicação em revista.

A organização deste artigo é a seguinte: $\mathrm{Na}$ Seção 2 serão introduzidos os conceitos básicos sobre álgebra max-plus, séries de potência e equações de datadores. Na Seção 3 são tratados os métodos de controle do fluxo de entrada de sistemas modelados por grafos de eventos temporizados, e finalmente, na Seção 4 a conclusão deste artigo.

\section{Conceitos Básicos}

A álgebra max-plus é um exemplo de álgebra de dióides ou, na verdade, um semianel idempotente.

Definição 2.1 Um semianel é um conjunto não vazio $S$ dotado de duas operações binárias $\oplus e \otimes$ tal que:

$\oplus$ é associativa e comutativa com elemento nulo $\varepsilon ; \otimes$ é associativa, distributiva sobre $\oplus$, e tem elemento unitário e; $\varepsilon$ é absorvente para $\otimes$.

Um dióide é completo se ele for fechado em relação a somas infinitas e se o produto for distributivo em relação a somas infinitas. A estrutura $(\mathbb{Z} \cup$ $\{-\infty\} \cup\{\infty\}, \max ,+)$ é um dióide completo usualmente denominado max-plus e notado por $\overline{\mathbb{Z}}_{\text {max }}$.
Definição 2.2 Idempotência e Ordem: Se em um semi-anel $S$ a soma $\oplus$ é idempotente, ou seja $a \oplus a=a, \quad \forall a \in S$, então é dito que $S$ é um semi-anel idempotente ou um dióide.

Quando a soma $\oplus$ é idempotente, fica imediatamente associado a ela uma relação de ordem:

$a=a \oplus b \Longleftrightarrow \exists c: a=b \oplus c, \forall a, b \quad$ e $c \in S$.

Definição 2.3 $O$ símbolo $\mathbb{Z}_{\max }$ denota o conjunto $\mathbb{Z} \cup\{-\infty\}$, com $\max e+$ sendo as duas operações binárias $\oplus e \otimes$, respectivamente.

Estas afirmações equivalentes definem uma relação de ordem (parcial) $\succeq$ em $S$ que está definido por $a \succeq b \Longleftrightarrow a=a \oplus b$.

Uma série é definida como: $s=\oplus_{k \in \mathbb{Z}} s(k) \gamma^{k} \in$ $\overline{\mathbb{Z}}_{\text {max }}[\gamma]$, a variável $\gamma$ pode ser interpretada como um operador de retardo.

Sejam $s_{1} \mathrm{e} \quad s_{2}$ duas séries periódicas de $\overline{\mathbb{Z}}_{\max }[\gamma]$. Então, $s_{1} \oint s_{2}$ é também uma série periódica.

\subsection{Equações por meio de Datadores}

A álgebra max-plus permite descrever a evolução da data de ocorrência dos eventos sujeitos a restrições de sincronização e de atraso por meio de equações de datadores.

A evolução dos sistemas são representados por equações lineares do tipo: $x(k)=A x(k-1) \oplus$ $B u(k)$. O vetor $x(k)$ representa o estado do sistema e $u(k)$ uma entrada externa. $A \geq I$, pois $x(k+1) \geq x(k)$, isto é, as datas de disparo das transições são não-decrescentes. A matriz $I$ é a matriz identidade sendo definida por $i_{i j}=e$ para $i=j$ e $i_{i j}=\varepsilon$ para $i \neq j$.

Os datadores são associados com cada transição. Para uma transição chamada $x_{i}$, a variável correspondente $x_{i}(k)$ será interpretada como o instante no qual ocorrerá o $k$-ésimo disparo da mesma. Desde o começo da simulação os disparos sucessivos de uma transição são numerados de maneira sequencial, a partir de uma origem universal (geralmente zero, ou poderia ser negativo). Logo, a função $k \mapsto x_{i}(k)$ não é decrescente (devido a que vários disparos podem ocorrer simultaneamente, pode não ser estritamente crescente). O tempo é medido em escala inteira, por isto, $x_{i}(k) \in \mathbb{Z}$.

As fichas iniciais existentes nos lugares do sistema não precisam cumprir os tempos de permanência exigidos para habilitar as transições, estes tempos já foram cumpridos, essas fichas foram disponibilizadas no tempo $-\infty$.

Considere uma linha de produção formada por uma única máquina. Essa máquina só pode processar um produto por vez. A matéria-prima é alimentada por uma transição de entrada $u_{1}$, e a 
saída do sistema é a transição $x_{2}$. O sistema é ilustrado pela Fig. (1).

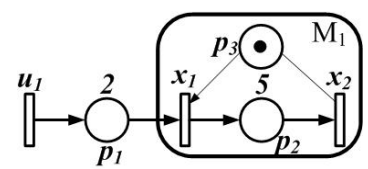

Fig. 1: Sistema de manufatura modelado por GET.

As transições disparam imediatamente após serem habilitadas, e tendo em conta que não existe conflito, é possível determinar quando ocorrerão os disparos das transições internas e de saída. As equações de datadores são obtidas de forma recursiva. A transição $u_{1}$ está sempre habilitada, injetando matéria-prima no sistema. O disparo de $x_{1}$ depende do disparo das transições $x_{2}$ e $u_{1}$. O disparo da transição $x_{2}$ é dito ocorrer no tempo $-\infty$, a ficha no lugar $p_{3}$ é uma condição inicial do sistema. O tempo de permanência da ficha no lugar $p_{3}$ é igual a zero. O disparo de $x_{1}$ ocorre após $u_{1}$ ter disparado e colocado uma ficha no lugar $p_{1}$, esta ficha ter cumprido o tempo de permanência deste local, de tal forma que: $x_{1}(k)=\max \{2+$ $\left.u_{1}(k), x_{2}(k-1)\right\}$. A próxima equação a ser obtida é de $x_{2}$. O disparo de $x_{2}$ ocorre após a máquina executar a sua operação, ou seja, após o disparo de $x_{1}$ mais o tempo de permanência da ficha no lugar $p_{2}$. Assim, $x_{2}(k)=x_{1}(k)+5$. A saída do sistema é $y(k)=x_{2}(k)$. Dessa forma, estão formadas as equações por datadores do sistema, Então:

$$
\begin{aligned}
x_{1}(k) & =2 u_{1}(k) \oplus x_{2}(k-1) \\
x_{2}(k) & =5 x_{1}(k) \\
y(k) & =x_{2}(k)
\end{aligned}
$$

\subsection{Operações com Matrizes}

Seja $S$ um dióide "escalar" arbitrário. Consideremos o conjunto $S^{n \times n}$ formado por todas as matrizes quadradas $n \times n$ com elementos em $S$. Definese sobre este conjunto a soma e a multiplicação convencionais a partir das operações soma $\oplus$ e multiplicação $\otimes$ de $S$, ou seja:

$$
\begin{gathered}
{[A \oplus B]=A_{i j} \oplus B_{i j}} \\
{\left[A_{i j} \otimes B_{i j}\right]=\bigoplus_{k}\left(A_{i k} \otimes B_{k j}\right)} \\
i=1, \ldots, n, j=1, \ldots, n, \forall A, B \in S^{n \times n} .
\end{gathered}
$$

Teorema 2.1 Considere o dióide $S$ completo $e$ $S^{n \times n}$ o dióide das matrizes com elementos em $S$ $e$ ainda as matrizes $A$ e $B \in S^{n \times n}$. O supremo da inequação $A X \preceq B$ existe e é dado pela matriz $L_{A}^{\sharp}(B) \in S^{n \times n}$, também representada por $A ф B$. Os elementos dessa matriz são calculados por:

$$
(A \phi B)_{i j}=\bigwedge_{l=1}^{n} A_{l i} \phi B_{l j} .
$$

$\wedge$ denota o maior inferior vinculado entre os elementos. $\oint$ denota uma operação de residuação.

\subsection{Estabilidade de GET's}

A estabilidade de um GET está relacionada ao número de fichas de um lugar. O GET é estável se o número de fichas é sempre limitado em todos os lugares internos.

A estabilidade de um GET só pode ser assegurada se todas as componentes funcionam a uma mesma taxa de produção, caso contrário haverá acumulação de fichas nos lugares de entrada do GET. Em sistemas de manufatura a taxa de produção é determinada pela quantidade de produtos que são produzidos pela unidade de tempo, tal que:

$$
\text { Taxa de Produção }=\frac{1}{\lambda}
$$

Sendo $\lambda$ o autovalor da matriz $A$ do sistema. $\lambda$ também é chamado de taxa de produção, no sentido de referir-se ao intervalo de tempo de saída de cada produto acabado do sistema produtivo operando em regime permanente.

\subsection{Função de Transferência}

Pode-se representar um datador $x(k)(k \in \mathbb{Z})$ por meio de uma transformada conhecida por transformada- $\gamma$, sendo formalmente definida como uma série de potências $X(\gamma)=\oplus_{k \in \mathbb{Z}} x(k) \gamma^{k}$. A variável $\gamma$ também pode ser considerada como o operador de atraso no domínio do evento (formalmente, $\gamma x(k)=x(k-1))$. Assim, pode-se expressar o comportamento de um GET em uma série de potências. Este dióide é representado na literatura por $\overline{\mathbb{Z}}_{\max }[\gamma]$

Por exemplo, considerando a seguinte equação de datadores de $x_{1}, x_{2}$ e $x_{3}$, tal que: $x_{3}(k)=$ $3 x_{1}(k-1) \oplus 8 x_{2}(k) \oplus 2 x_{3}(k-1)$. Então, a sua respectiva transformada- $\gamma$ será $x_{3}(\gamma)=3 \gamma x_{1}(\gamma) \oplus$ $8 x_{2}(\gamma) \oplus 2 \gamma x_{3}(\gamma)$. Consequentemente, para esse conjunto de equações, será obtida a seguinte representação matricial: $X=A X \oplus B U$ e $Y=C X$. A relação de transferência entre entrada e saída pode ser expressa por $Y=H U$, sendo $H=C A^{*} B$ a função de transferência, tal que $H \in \overline{\mathbb{Z}}_{\max }[\gamma]^{p \times m}$.

\subsubsection{Controle $P-F$}

As principais vantagens dessa abordagem são que ela garante otimalidade em relação aos estoques que alimentam a planta para qualquer modelo de referência escolhido (Maia et al., 2005), a ação de controle ótima é sempre máxima e não há nenhuma restrição na escolha do modelo de referência. A Fig. (2) ilustra o controle. Dessa forma, escrevem-se as seguintes equações: $u=P v \oplus P F y$, $y=(H P F) y \oplus H P v, G_{c} v=H P(F H P) * v$, e $G_{u v}=P(F H P) * v$, sendo $G_{c}$ e $G_{u v}$ as funções 


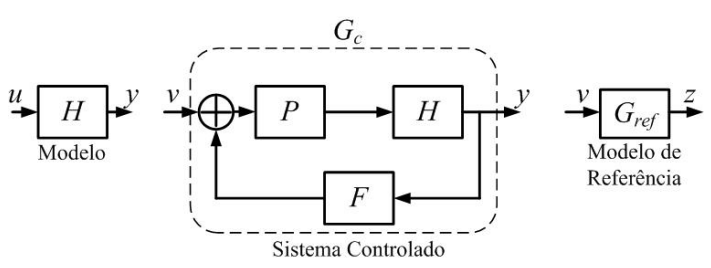

Fig. 2: Controle $P-F$.

de transferência em malha fechada entre $y$ e $v$ e entre $u$ e $v$, respectivamente.

Dessa forma, é possível encontrar as matrizes do controlador $P$ e $F$ que asseguram a maior função de transferência entre $u$ e $v$, tal que: $G_{c} \preccurlyeq$ $G_{r e f}$.

O problema de otimização proposto tem sempre solução ótima que é obtida com:

$$
\begin{aligned}
P_{o p} & =H \phi G_{r e f} . \\
F_{o p} & =\left(H P_{o p}\right) \phi\left(H P_{o p}\right) \phi\left(H P_{o p}\right) .
\end{aligned}
$$

\section{Métodos de Controle do Fluxo de Entrada}

Nesta seção serão calculados os controladores do fluxo de entrada de alimentação para sistemas de manufatura modelados por GET. No primeiro exemplo, um controlador é obtido para controlar o fluxo de entrada de um sistema com várias entradas e uma única saída utilizando o modelo de referência, baseado no dióides de série $\overline{\mathbb{Z}}_{\max }[\gamma]$. Logo após, é aplicado o método do caminho crítico para o mesmo sistema. O objetivo é comparar os resultados obtidos pelos dois métodos. Em seguida, o método do caminho crítico é aplicado para sistemas com múltiplas entradas e múltiplas saídas.

No final da seção, é apresentado um método para calcular os parâmetros de um controlador que controla os disparos de duas transições internas de um sistema modelado por GET, com o objetivo de atender a condições especiais de restrições do processo. Este tipo de controle é realizado utilizandose a base em dióides $\overline{\mathbb{Z}}_{\text {max }}$, e que, até o presente momento, não foi abordado utilizando-se séries de potência $\left(\overline{\mathbb{Z}}_{\max }[\gamma]\right)$.

\subsection{Controle por Modelo de Referência}

O objetivo deste trabalho não é detalhar com precisão os processos de cálculo ${ }^{1}$ para obtenção do controlador por modelo de referência. Uma rápida síntese de um controlador utilizando o modelo de referência será descrita utilizando-se como exemplo o GET representado pela Fig. (3).

Este modelo representa um pequeno processo com três entradas de alimentação, três estoques

\footnotetext{
${ }^{1}$ Os resultados do modelo de referência encontrados foram obtidos por meio de programas em $C++$ e Scilab obtidos em http://193.49.146.171/ hardouin/.
}

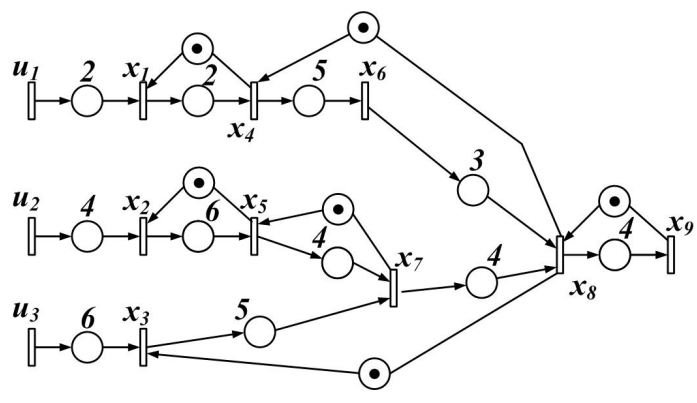

Fig. 3: Sistema de manufatura modelado por GET.

de entrada, oito postos de trabalho e uma única saída. As entradas de alimentação estão descritas pelas transições $u_{1}, u_{2}$ e $u_{3}$. O objetivo é calcular a maior realimentação de saída realizável no controle de realimentação de saída para entrada $\left(G_{\text {ref }}\right)$.

A função de transferência é dada por ${ }^{2}$ :

$H=\left(\begin{array}{lll}16 \gamma^{3}(8 \gamma)^{*} & 22(6 \gamma)^{*} & \left.\left.19 \gamma^{(9 \gamma}\right)^{*}\right) .\end{array}\right.$

Com o objetivo de superar um problema de instabilidade que surge nos caminhos de $u_{1}, u_{2} \mathrm{e}$ $u_{3}$ até a saída, devido as diferenças das taxas de produção, que são mostradas pela função de transferência $H, u_{1}$ até $y, \frac{1}{8}$ ficha/unidade de tempo, do caminho $u_{2}, \frac{1}{6}$ ficha/unidade de tempo, e o do caminho $u_{3}, \frac{1}{9}$ ficha/unidade de tempo. Portanto, devido à estrutura do processo, uma instabilidade surge assim como demasiado muitas partes são admitidas simultaneamente nas entradas $u_{1}$ e $u_{2}$ por causa das diferenças das taxas de produção dos postos de trabalho, a marcação dos lugares localizado nesses caminhos cresce sem limites. Assim, um objetivo aqui é impor, com ajuda do controlador, uma taxa de produção do caminho do gargalo $u_{3}$ para todo o sistema. Então, baseados nos resultados da função de transferência e nos valores das taxas dos caminhos, será definido:

$$
G_{r e f}=\left(\begin{array}{lll}
22 \gamma^{3}(9 \gamma)^{*} & 22(9 \gamma)^{*} & 22 \gamma(9 \gamma)^{*}
\end{array}\right),
$$

que satisfaz a todas as restrições dos caminhos. Dessa forma, a síntese do controlador é obtida por:

$P_{o p}=(9 \gamma)^{*}\left(\begin{array}{ccc}3 & 3 & 3 \\ e & e & e \\ 6 & 6 & 6\end{array}\right), F_{o p}=\left(5 \gamma^{3}\right)^{*}(9 \gamma)^{*}\left(\begin{array}{l}e \\ e \\ e\end{array}\right)$.

A realização desse controlador ótimo é ilustrado na Fig. (4).

Os tempos de saída dos produtos são:

Tabela 1: Tempos de Saída

\begin{tabular}{|c|c|c|c|c|c|c|c|c|}
\hline Saída & \multicolumn{7}{|c|}{ Tempos(seg) } \\
\hline$y$ & 22 & 31 & 40 & 49 & 58 & 67 & 76 & 85 \\
\hline
\end{tabular}

\footnotetext{
${ }^{2}$ Resultados encontrados utilizando-se programas em $C++$ e Scilab obtidos em http://193.49.146.171/ hardouin/.
} 


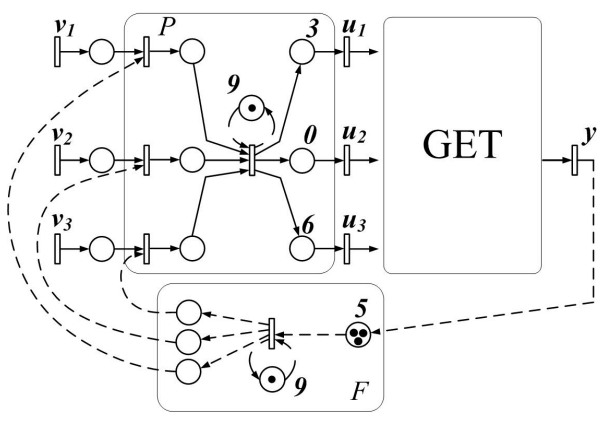

Fig. 4: Controlador em linhas tracejadas.

\subsection{Método do Caminho Crítico}

Na utilização do método do caminho crítico, a matriz $A$ será descrita de forma diferente da utilizada no método pelo modelo de referência, tal que:

$x(k)=A_{0} x(k) \oplus A_{1} x(k-1) \oplus B_{0} u(k) ;$

$y(k)=C_{0} x(k)$.

equivalente representado por:

$x(k)=A_{0}^{*} A_{1} x(k-1) \oplus A_{0}^{*} B_{0} u(k) ;$

$y(k)=C x(k)$.

sendo: $A_{0}^{*}=I \oplus A_{0} \oplus A_{0}^{2} \oplus A_{0}^{3} \oplus$. . ( $I$ é a matriz identidade e o operador $(*)$ é chamado estrela de Kleene. Demonstração pode ser encontrada em (Baccelli et al., 1992)).

Para o exemplo da Fig. (3), a modelagem matricial é dada por:

$A_{0}=\left[\begin{array}{lllllllll}\varepsilon & \varepsilon & \varepsilon & \varepsilon & \varepsilon & \varepsilon & \varepsilon & \varepsilon & \varepsilon \\ \varepsilon & \varepsilon & \varepsilon & \varepsilon & \varepsilon & \varepsilon & \varepsilon & \varepsilon & \varepsilon \\ \varepsilon & \varepsilon & \varepsilon & \varepsilon & \varepsilon & \varepsilon & \varepsilon & \varepsilon & \varepsilon \\ 2 & \varepsilon & \varepsilon & \varepsilon & \varepsilon & \varepsilon & \varepsilon & \varepsilon & \varepsilon \\ \varepsilon & 6 & \varepsilon & \varepsilon & \varepsilon & \varepsilon & \varepsilon & \varepsilon & \varepsilon \\ \varepsilon & \varepsilon & \varepsilon & 5 & \varepsilon & \varepsilon & \varepsilon & \varepsilon & \varepsilon \\ \varepsilon & \varepsilon & 5 & \varepsilon & 4 & \varepsilon & \varepsilon & \varepsilon & \varepsilon \\ \varepsilon & \varepsilon & \varepsilon & \varepsilon & \varepsilon & 3 & 4 & \varepsilon & \varepsilon \\ \varepsilon & \varepsilon & \varepsilon & \varepsilon & \varepsilon & \varepsilon & \varepsilon & 4 & \varepsilon\end{array}\right] B_{0}=\left[\begin{array}{lll}2 & \varepsilon & \varepsilon \\ \varepsilon & 4 & \varepsilon \\ \varepsilon & \varepsilon & 6 \\ \varepsilon & \varepsilon & \varepsilon \\ \varepsilon & \varepsilon & \varepsilon \\ \varepsilon & \varepsilon & \varepsilon \\ \varepsilon & \varepsilon & \varepsilon \\ \varepsilon & \varepsilon & \varepsilon \\ \varepsilon & \varepsilon & \varepsilon\end{array}\right]$

$A_{1}=\left[\begin{array}{lllllllll}0 & \varepsilon & \varepsilon & 0 & \varepsilon & \varepsilon & \varepsilon & \varepsilon & \varepsilon \\ \varepsilon & 0 & \varepsilon & \varepsilon & 0 & \varepsilon & \varepsilon & \varepsilon & \varepsilon \\ \varepsilon & \varepsilon & 0 & \varepsilon & \varepsilon & \varepsilon & \varepsilon & 0 & \varepsilon \\ \varepsilon & \varepsilon & \varepsilon & 0 & \varepsilon & \varepsilon & \varepsilon & 0 & \varepsilon \\ \varepsilon & \varepsilon & \varepsilon & \varepsilon & 0 & \varepsilon & 0 & \varepsilon & \varepsilon \\ \varepsilon & \varepsilon & \varepsilon & \varepsilon & \varepsilon & 0 & \varepsilon & \varepsilon & \varepsilon \\ \varepsilon & \varepsilon & \varepsilon & \varepsilon & \varepsilon & \varepsilon & 0 & \varepsilon & \varepsilon \\ \varepsilon & \varepsilon & \varepsilon & \varepsilon & \varepsilon & \varepsilon & \varepsilon & 0 & 0 \\ \varepsilon & \varepsilon & \varepsilon & \varepsilon & \varepsilon & \varepsilon & \varepsilon & \varepsilon & 0\end{array}\right]$

O método propõe um controlador que atrasa ao máximo as entradas de matéria-prima em uma planta, garantindo uma dada taxa de produção $\beta$, controlando os instantes de disparos das transições de entrada, atuando segundo a seguinte lei de controle:

$$
\begin{gathered}
u(k)=F w(k) \\
w(k)=G w(k-1) \oplus L y(k-m)
\end{gathered}
$$

sendo $F, G$ e $L$ matrizes de dimensões apropriadas. $F$ é a matriz que contém os tempos de atrasos das entradas do sistema, os elementos dessa matriz são representados pelo vetor $[\varphi]^{1 \times l} ; G$ é a taxa de disparo do controlador, como o sistema tratado possui uma única saída, então é necessário uma única taxa de disparo para o controlador, e será denominada por $\beta$; $L$ é uma matriz que representa um atraso da informação após a saída do produto, para o caso tratado neste trabalho não existe atraso de informação, então a matriz $L$ será considerada uma matriz identidade e $m$ é o número de fichas do controlador. O controlador de realimentação é ilustrado na Fig. (7).

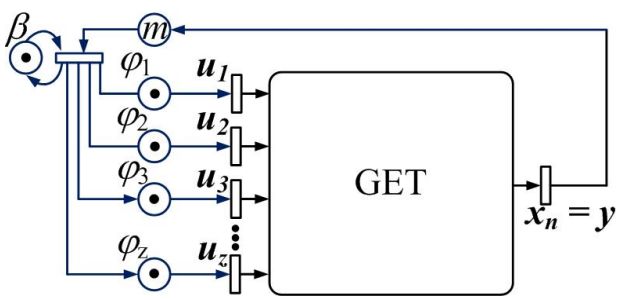

Fig. 5: Sistema de Realimentação para Estoque Otimizado das Entradas.

\subsubsection{Cálculo dos parâmetros do Contro- lador}

O método do caminho crítico consiste em determinar os atrasos máximos que as entradas de alimentação podem ter, de forma a não interferir nos tempos de saída dos produtos. O método calcula o maior tempo de percurso (caminho crítico) que uma ficha fará dentro do sistema, e obtém as diferenças de tempo entre o tempo do caminho crítico e todos os maiores caminhos de cada entrada até a saída. Os valores obtidos são os atrasos que as entradas poderão ter sem atrasar a saída do sistema.

$$
[\varphi]_{1 \times l}=C B \phi \delta .
$$

O comprimento do caminho crítico $(\delta)$ é dado por:

$$
\delta=\bigoplus C B
$$

O número de fichas do controlador $(m)$ é o número de disparos necessários que o controlador precisa efetuar para atuar independente do sinal de saída do sistema. O valor de $m$ é o menor inteiro maior que a divisão do comprimento do caminho crítico $(\delta)$ pelo valor da taxa de saída do sistema $(\lambda)$, tal que:

$$
m=\left\lceil\frac{\delta}{\lambda}\right\rceil
$$

O valor da taxa de disparo do controlador do sistema de realimentação $(\beta)$ deverá ser igual à taxa de saída do sistema $(\lambda), \beta=\lambda$, tal que:

$$
\lambda=\bigoplus_{j=1}^{n}\left(\operatorname{traço}\left(A^{j}\right)\right)^{1 / j}
$$

\subsubsection{Resultado}

Parâmetros do controlador encontrados (Fig. 6) aplicando-se o método do caminho crítico no sistema ilustrado na Fig. 3. : 


$$
\beta=9, \varphi=\left[\begin{array}{lll}
6 & 0 & 3
\end{array}\right] \text { e } m=3 \text {. }
$$

Sem o controle proposto, as entradas de alimentação injetariam material no processo produtivo de forma exagerada, gerando instabilidade no sistema.

Os tempos de saída dos produtos do sistema são: $y(1)=22, y(2)=31, y(3)=40, y(4)=49$, $y(5)=58 \ldots$

O sistema de controle proposto atrasa a entrada de matéria-prima, preservando a taxa de produção do sistema.

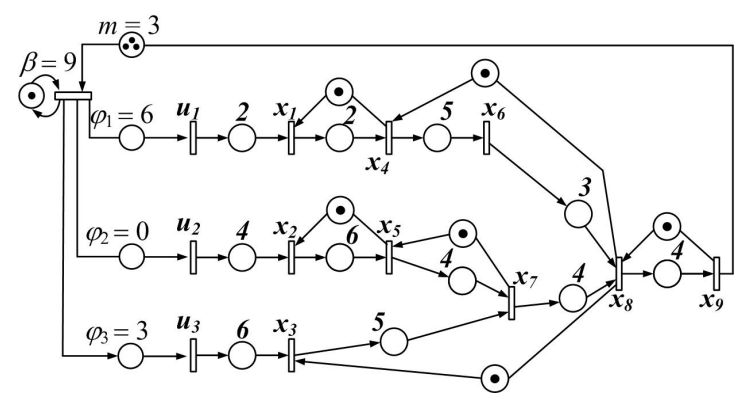

Fig. 6: Sistema de realimentação aplicando-se o método do caminho crítico.

Os tempos de saída dos produtos são:

Tabela 2: Tempos de Saída

\begin{tabular}{|c|c|c|c|c|c|c|c|c|}
\hline Saída & \multicolumn{10}{|c|}{ Tempos(seg) } \\
\hline$y$ & 22 & 31 & 40 & 49 & 58 & 67 & 76 & 85 \\
\hline
\end{tabular}

Os resultados são iguais, considerando-se a taxa de saída dos produtos e os tempos de atrasos encontrados para as entradas do sistema, mas o método do caminho crítico é mais simples de ser calculado e oferece uma estrutura de menor complexidade quando comparado com o modelo de referência. Mais opções de controle podem ser executadas utilizando-se a estrutura $\overline{\mathbb{Z}}_{\text {max }}$, como será desenvolvido em seguida.

\subsection{Método do Caminho Crítico aplicado em sis- temas MIMO}

A aplicação do método do caminho crítico para sistemas com múltiplas entradas e múltiplas saídas (MIMO-multiple-input and multiple-output) adotará a estrutura de controle ilustrada na Fig. 7, em malha aberta.

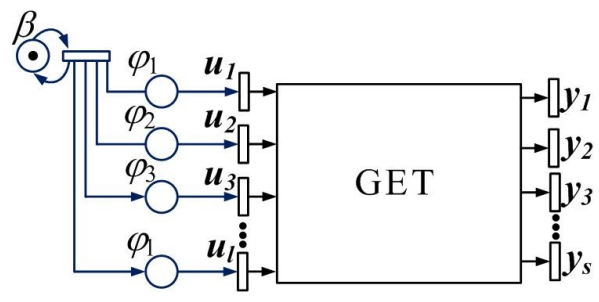

Fig. 7: Controle em Malha Aberta.

\subsubsection{Exemplo Ilustrativo}

Considere um sistema MIMO consistindo de cinco entradas $\left(u_{1}, u_{2}, u_{3}, u_{4}, u_{5}\right)$, dezesseis transições $\operatorname{internas}\left(x_{1}, x_{2}, x_{3}, \ldots, x_{16}\right)$, vinte estações de trabalho(as indicações das estações serão omitidas para facilitar a visualização do desenho) e três saídas $\left(y_{1}, y_{2}, y_{3}\right)$ (Fig. 8).

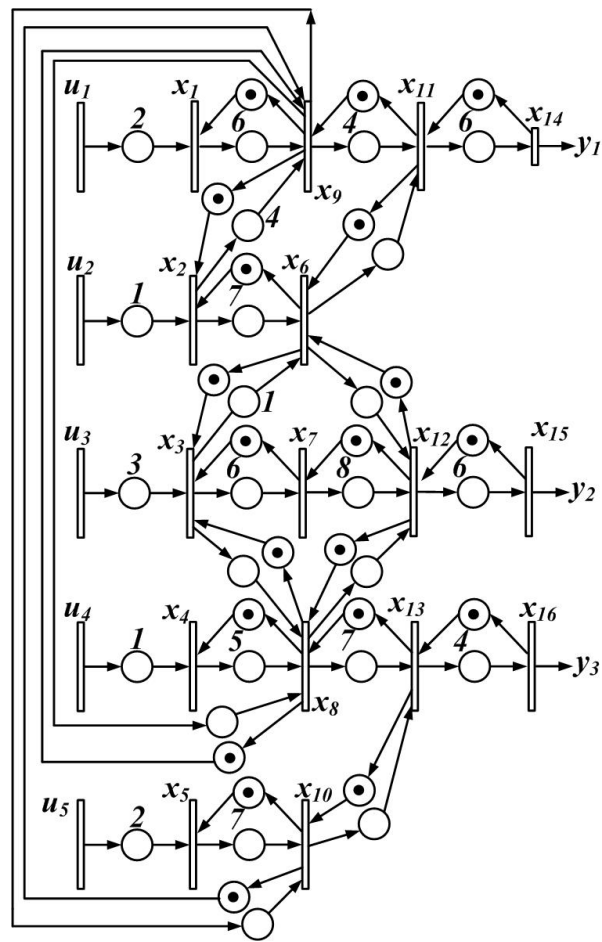

Fig. 8: Sistema de Manufatura.

O objetivo deste exemplo é o de encontrar o maior atraso para cada entrada, sem comprometer a demanda de produção. Para resolver o problema, será aplicado o método do caminho crítico para encontrar os parâmetros do controlador que garantem o melhor controle dos disparos das transições de entrada. As equações recursivas e a modelagem matricial serão omitidas (para não ultrapassar o número de páginas permitido), mas podem ser obtidas por meio de análise do GET.

Os tempos de saída dos produtos são:

Tabela 3: Tempos das Saídas

\begin{tabular}{|c|c|c|c|c|c|c|c|c|}
\hline Saídas & \multicolumn{10}{|c|}{ Tempos(seg) } \\
\hline$y_{1}$ & 17 & 26 & 35 & 44 & 53 & 62 & 71 & 80 \\
\hline$y_{2}$ & 17 & 25 & 33 & 42 & 51 & 60 & 69 & 78 \\
\hline$y_{3}$ & 15 & 24 & 33 & 42 & 51 & 60 & 69 & 78 \\
\hline
\end{tabular}

A taxa de saída dos produtos é única, $\lambda_{1}=9$.

\subsubsection{Resultado}

Os seguintes parâmetros do controlador foram encontradas pela aplicação do método do caminho 
crítico, resultando na estrutura ilustrada na Fig. 9:

$$
\beta=9 \text { е } \varphi=\left[\begin{array}{lllll}
3 & 6 & 0 & 0 & 6
\end{array}\right]
$$

Sem o controle proposto, as entradas injetariam material no processo de produção até a saturação das entradas, provocando a instabilidade do sistema.

O sistema de controle proposto garante o máximo atraso possível das matérias-primas nas entradas do sistema, preservando o ritmo de produção apropriado, evitando o estouro dos estoques do sistema.

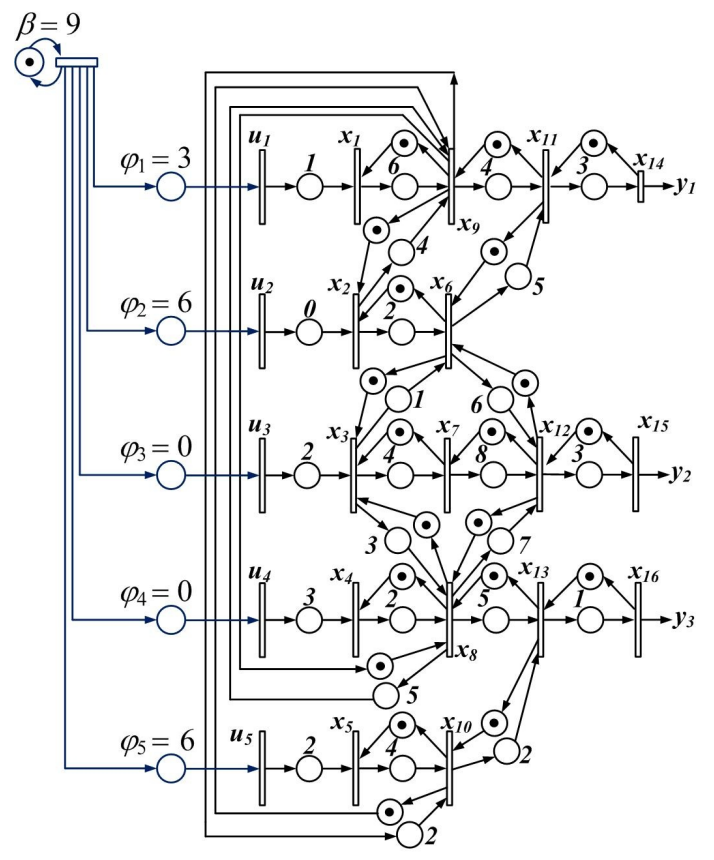

Fig. 9: Controle Implementado Utilizando-se o Método do Caminho Crítico.

\subsection{Sincronização do Disparo de Transições}

Considere três máquinas $\left(M_{1}, M_{2}\right.$ e $\left.M_{3}\right)$ de um processo produtivo, as máquinas $M_{1}$ e $M_{2}$ produzem partes de peças de um produto final que deverão ser acopladas na máquina $M_{3}$. Para realizar o acoplamento, a máquina $M_{1}$ injeta uma cola(adesivo) de secagem rápida na peça 1 e a máquina $M_{2}$ prepara a uma peça 2 que será unida a peça de número 1 na máquina $M_{3}$. $\mathrm{O}$ adesivo colocado na peça número 1 possui um tempo de cura muito rápido e muitas das peças podem ser perdidas pelo fato de o processo não liberar as peças no mesmo instante de tempo para que sejam unidas imediatamente na máquina $M_{3}$. O ajuste do processo precisa garantir que não exista atraso entre os instantes em que as peças são disponibilizadas para a máquina $M_{3}$. Para satisfazer tal restrição e não comprometer os estoques de entradas, o controle será feito a partir da implementação de atrasos nas entradas de material no processo, garantindo que com esses atrasos as máquinas $\left(M_{1} \mathrm{e}\right.$
$M_{2}$ ) terminem suas operações no mesmo instante de tempo. Um nova taxa de produção é determinada para garantir o ajuste do processo, caso esta taxa seja diferente da taxa de produção do sistema o maior valor é considerado, esse nova taxa é o valor do disparo do controlador realimentado.

O método consiste em sincronizar o disparo de duas transições em um GET. Para isso, é calculado o maior tempo que duas transições precisam para estarem novamente habilitadas para efetuarem um disparo sincronizado, aplicando-se novos tempos de atraso na entrada do sistema (matriz $F$ do controlador) e uma nova taxa de produção do sistema ( $\beta$ do controlador). O objetivo é determinar o melhor ritmo de produção do sistema de manufatura para atender a condição de restrição.

\subsubsection{Cálculo dos parâmetros de controla- dor}

Utilizando-se a mesma estrutura de controle (Fig. 7), os parâmetros do controlador para efetuar o controle do disparo de duas transições internas de um sistema são calculados da seguinte forma:

A taxa de disparo do controlador $(\beta)$ é dada por:

$\beta=\bigoplus_{q=1}^{l}\left([A B]_{r q} \oplus[A B]_{s q}\right) \otimes\left(-\left([B]_{r q} \oplus[B]_{s q}\right)\right)$,

para $q=1,2, \ldots, l$, sendo $l$ o número de entradas do sistema, $n$ o numero de transições internas do sistema, os índices $r$ e $s$ correspondente aos índices das duas transições que se deseja controlar $\left(x_{r}\right.$ e $\left.x_{s}\right)$. Sendo:

Os tempos de atrasos acrescentados antes das entradas de alimentação, no controlador, e representados por $\varphi_{q}$, elementos da matriz $F$, são os valores máximos de atrasos, que somados aos atrasos já existentes nos maiores caminhos entre cada entrada de alimentação $\left(u_{q}\right)$ até as transições a serem controladas $\left(x_{r}\right.$ e $\left.x_{s}\right)$, tornam esses caminhos iguais, ou seja, com o mesmo valor de tempo de atraso. Tal que:

$$
F=[\varphi]_{1 \times l}^{T}=\rho_{u r / s} \otimes\left[\begin{array}{c}
-\left([B]_{r 1} \oplus[B]_{s 1}\right) \\
-\left([B]_{r 2} \oplus[B]_{s 2}\right) \\
\vdots \\
-\left([B]_{r l} \oplus[B]_{s l}\right)
\end{array}\right]
$$

sendo $\rho_{u r / s}$ o maior dos somatórios dos tempos de atraso dos caminhos desde as entradas até as transições $x_{r}$ e $x_{s}$.

O número de fichas do controlador é calculado por:

$$
m=\left\lceil\frac{\delta}{\beta}\right\rceil
$$

\subsubsection{Exemplo Ilustrativo}

Considere o sistema de manufatura proposto na Figura (3). O objetivo é calcular a que taxa o sistema deverá operar para garantir a restrição de 
controle $x_{4}(k)=x_{7}(k)$. É possível verificar que esse tipo de controle restringirá a uma única peça em dois postos de trabalhos consecutivos. A modelagem matricial é mesma utilizada no método do caminho crítico.

Cálculo dos Parâmetros do Controlador para controle de restrição: $x_{4}(k)=x_{7}(k)$

Taxa de disparo do controlador $(\beta): \beta=\psi \oplus$ $\lambda=13 \oplus 9=13$

Tempos de atrasos das entradas do sistema $[\varphi]_{1 \times l}: \varphi=\left[\begin{array}{lll}10 & 0 & 3\end{array}\right]$

Número de fichas do controlador $(m): m=$ $\left\lceil\frac{\delta}{\lambda}\right\rceil=\left\lceil\frac{22}{13}\right\rceil=2$

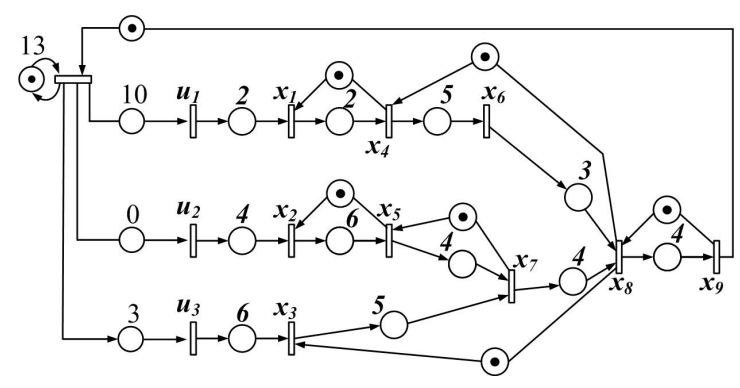

Fig. 10: Resultado: controle de disparo de transições.

\section{Conclusão}

O método do caminho crítico desenvolvido garante o melhor controle possível para o disparo das transições de entrada do sistema. Para a classe de problemas estudados, é mais vantajoso trabalhar com a base de dióides $\mathbb{Z}_{\max }$ do que com a base na série dióides $\mathbb{Z}_{\max }(\gamma)$, pela simplicidade na implementação do sistema de controle. Os parâmetros são encontrados sem a necessidade do uso de algoritmos.

Embora o método do caminho crítico seja mais simples de ser empregado, este ainda é restrito e aplicado a somente uma classe de sistemas modelados por GET, enquanto que o método pelo modelo de referência é mais abrangente na análise de sistemas max-plus lineares. Entretanto, nesse caso deve-se: 1) trabalhar com condições iniciais canônicas; 2) o controle é just-in-time em um contexto no qual há comparação de funções de transferências, o que é diferente quando se compara diretamente a saída do sistema; e 3 ) não há método para a escolha da função de transferência.

O controle de disparo de duas transições internas do sistema oferece uma variedade ampla de novas possibilidades de controle, é possível por meio desta técnica dimensionar a real capacidade de produção para atender as condições especiais de restrições. Restrições desse tipo ainda não são tratadas por meio de séries de potência, particularmente, por nenhum modelo de referência.

Trabalhos futuros serão desenvolvidos para ampliar a análise do método do caminho crítico para outros sistemas modelados por GET, com restrições no estado e no controle, e ainda, com perspectivas na análise de falhas em sistemas a eventos discretos modelados por GETs.

\section{Agradecimentos}

Os autores agradecem o apoio da FAPEAM, FAPEMIG, CNPq e CAPES para o desenvolvimento deste trabalho. O primeiro autor foi bolsista da FAPEAM no Programa de Apoio à Formação de Recursos Humanos Pós-Graduados do Estado do Amazonas, Doutorado Interinstitucional.

\section{Referências}

Baccelli, F., Cohen, G., Olsder, G. J. and Quadrat, J. P. (1992). Synchronization and Linearity, Wiley.

Boimond, J. L. and Ferrier, J. L. (1996). Internal model control and max-algebra: controller design, IEEE Transactions on Automatic Control 41(3): 457-461. DOI $10.1109 / 9.486651$

Brunsch, T., Raisch, J. and Hardouin, L. (2012). Modeling and control of high-throughput screening systems, Con-trol Eng. Practice 20(1): 14-23. DOI: 10.1016/j.conengprac.2010.12.006

Cassandras, C. G. and Lafortune, S. (1999). Introduction to Discrete Event Systems, Kluwer Academic Publishers. DOI: 10.1007/978-1-4757-4070-7

Cottenceau, B., Hardouin, L., Boimond, J.-L. and Ferrier, J.L. (1999). Synthesis of greatest linear feedback for timedevent graphs in dioid, IEEE Transactions on Automatic Control 44(6): 1258-1262. DOI: 10.1109/9.769386

Cottenceau, B., Hardouin, L., Boimond, J-L and Ferrier, JL. (2001). Model reference control for timed event graphs in dioids, Automatica 37(9): 1451-1458. DOI: 10.1016/S0005-1098(01)00073-5

Dias, J. R. S., Maia, C. A. and Jr, V. F. L. (2012). Método para controle de fluxo de entrada em sistemas max-plus lineares garantindo uma dada taxa de produção, Anais do XIX Congresso Brasileiro de Automática pp. 11571164

Maia, C. A. (2003). Identificação e controle de sistemas a eventos discretos na álgebra (max, + ), PhD thesis, UNICAMP.

Maia, C. A., Hardouin, L., Mendes, R. S. and Cottenceau, B. (2003). Optimal closed-loop control of timed event graphs in dioids, IEEE Transactions on Automatic Control 48(12): 2284-2287. DOI: 10.1109/TAC.2003.820666

Maia, C. A., Hardouin, L., Santos-Mendes, R, and Loiseau, J. J. (2011). A super-eigenvector approach to control constrained max-plus linear systems, 50th IEEE Conference on Decision and Control and European Control Conference. DOI: 10.1109/CDC.2011.6160411

Maia, C. A., Mendes, R. S., Luders, R. and Hardouin, L. (2005). Estratégias de controle por modelo de referência de sistemas a eventos discretos max-plus lineares, Revista Controle e Automação .

Menguy, E., Boimond, J. L., Hardouin, L. and Ferrier, J. L. (2000). Just-in-time control of timed event graphs: update of reference input, presence of uncontrollable input, IEEE Transactions on Automatic Control 45(11): 21552159. DOI: $10.1109 / 9.887652$

Murata, T. (1989). Petri nets: Properties, analysis and applications, Proc. of the IEEE 77(4): 541-580. DOI: $10.1109 / 5.24143$

Schutter, B. D. and van den Boom, T. (2000). Model predictive control for max-plus-linear systems, Proceedings of the American Control Conference, Vol. 6, pp. 4046-4050.

Zhu, Q., Sheng, W. and Xi, N. (2004). Max-plus algebra model for on-line task scheduling of a reconfigurable manufacturing work-cell, Proc. IEEE/RSJ International Conf. on Intelligent Robots and Systems, Vol. 2, pp. 1245-1250. 